\title{
A Path-Integral Approach to the Cameron-Martin-Maruyama-Girsanov Formula Associated to a Bilaplacian
}

\author{
Rémi Léandre ${ }^{1,2}$ \\ ${ }^{1}$ Institut de Mathématiques, Université de Bourgogne, 21000 Dijon, France \\ ${ }^{2}$ Laboratoire de Mathématiques, Université de Franche-Comté, Route de Gray, 25030 Besançon, France
}

Correspondence should be addressed to Rémi Léandre, remi.leandre@univ-fcomte.fr

Received 4 October 2012; Revised 28 November 2012; Accepted 28 November 2012

Academic Editor: Ti J. Xiao

Copyright (c) 2012 Rémi Léandre. This is an open access article distributed under the Creative Commons Attribution License, which permits unrestricted use, distribution, and reproduction in any medium, provided the original work is properly cited.

We define the Wiener product on a bosonic Connes space associated to a Bilaplacian and we introduce formal Wiener chaos on the path space. We consider the vacuum distribution on the bosonic Connes space and show that it is related to the heat semigroup associated to the Bilaplacian. We deduce a Cameron-Martin quasi-invariance formula for the heat semigroup associated to the Bilaplacian by using some convenient coherent vector. This paper enters under the Hida-Streit approach of path integral.

\section{Introduction}

Let us recall some basic tools of Wiener analysis. Let $B_{t}$ be a one-dimensional Brownian motion starting from 0 . It is classically related to the heat equation on $\mathbb{R}$ :

$$
\frac{\partial}{\partial t} E\left[f\left(B_{t}\right)\right]=\frac{1}{2} E\left[\Delta f\left(B_{t}\right)\right]
$$

where $\Delta=\partial^{2} / \partial x^{2}$ is the standard Laplacian and $f$ is a smooth function with bounded derivatives at each order. Associated to the heat equation there is a convenient probability measure on a convenient path space. Almost surely, the trajectory of $B$ is continuous. We construct by this way the Wiener measure $d P$ on the continuous path space endowed with its Borelian $\sigma$-algebra. Let $\mathbb{H}$ be the Hilbert space $L^{2}([0,1] ; \mathbb{R})$. We consider the symmetric tensor 
product $\mathbb{H}^{\widehat{\otimes} n}$ of this Hilbert space. It is constituted of maps $h_{n}\left(s_{1}, \ldots, s_{n}\right)$ symmetric in $s_{i}$ such that

$$
\left\|h_{n}\right\|^{2}=\int_{[0,1]^{n}} h_{n}^{2}\left(s_{1}, \ldots, s_{n}\right) d s_{1} \cdots d s_{n}<\infty
$$

We consider the symmetric Fock space $F(\mathbb{H})$ of set $\sigma=\sum_{n=0}^{\infty} h_{n}$ such that

$$
\|\sigma\|^{2}=\sum n !\left\|h_{n}\right\|^{2}<\infty
$$

We consider the vacuum expectation.

$$
\mu[\sigma]=h_{0} .
$$

With an element $h_{n}$ of $\mathbb{H}^{\otimes} \hat{n}$ is associated the Wiener chaos

$$
\Psi\left(h_{n}\right)=\int_{[0,1]^{n}} h_{n}\left(s_{1}, \ldots, s_{n}\right) d B_{S_{1}} \cdots d B_{s_{n}} .
$$

The mat $\Psi$ realizes a isomorphism between $F(\mathbb{H})$ and $L^{2}(d P)$. On the level of the Fock space some important elements are constituted by coherent vectors:

$$
\sigma=\sum \frac{h^{\otimes n}}{n !}
$$

The functional associated to such a coherent vector is a so-called exponential martingale

$$
\Psi(\sigma)=\exp \left[\int_{0}^{1} h_{s} d B_{s}-\frac{\|h\|^{2}}{2}\right] .
$$

We refer to the books of Hida et al. [1], to the book of Obata [2], and to the book of Meyer [3] for an extensive study on that subject. Especially on the Fock space, we can define the Wiener product:

$$
\Psi\left(\sigma_{1} \cdot \sigma_{2}\right)=\Psi\left(\sigma_{1}\right) \Psi\left(\sigma_{2}\right)
$$

where we consider the ordinary product of the two $\Psi\left(\sigma_{i}\right)$. For that, we use the Itô table for the Laplacian

$$
\begin{gathered}
d B_{s} \cdot d B_{s}=\frac{1}{2} d s, \\
d B_{s} \cdot d s=d s \cdot d s=0
\end{gathered}
$$


which reflect algebraically the Itô formula for the Brownian motion. From this Itô table, we deduce classically that if $\sigma$ is an exponential vector, $\Psi(\sigma)=\exp \left[\int_{0}^{1} h_{s} d B_{s}-\|h\|^{2} / 2\right]$ and not $\exp \left[\int_{0}^{1} h_{s} d B_{s}\right]$.

The law of $B_{t}+\int_{0}^{t} h_{s} d s$ is absolutely continuous with respect of the law of $B_{t}$, and the Radon-Nikodym derivative between these two laws is $\Psi(\sigma)=\exp \left[-\int_{0}^{1} h_{s} d B_{s}-\|h\|^{2} / 2\right]$. It is the subject of the Cameron-Martin formula.

The construction of a full path probability measure associated to a semi-group is related to Hunt theory: the generator $L$ of the semi-group has to satisfy maximum principle. We are motivated where we take others type of generator. To simplify the computations we take the simplest of such operators $L=-\partial^{4} / \partial x^{4}$. We have implemented recently some stochastic tools for semi-groups whose generators do not simplify maximum principle ([4$10])$. We construct in $[8,9]$ the Wiener distribution associated to a Bilaplacian using the Hida-Streit approach of path integrals as distribution. We refer to the works of Funaki [11], Hochberg [12], Krylov [13], and the review paper of Mazzucchi [14] for other approaches. We refer to the review paper of Albeverio [15] for various approach of path integrals.

In the Hida-Streit approach of path integral, there are basically 3 objects:

(i) an algebraic space, generally a kind of Fock space;

(ii) a map $\Psi$ from this algebraic space into a set of functionals on a mapping space;

(iii) the path integral is continuous on the level of the algebraic set. We say that it is an Hida-type distribution.

Generally, people were considering map $\Psi$ as the map Wiener chaos. A breakdown was performed by Getzler [16] motivated by the works of Atiyah-Bismut-Witten relating the structure of the free loop space and the Index theory. Developments were done by Leandre in $[17,18]$. Especially, in $[8,9]$ we were using map $\Psi$ as related to cylindrical functional to define a path integral associated to the Bilaplacian and to state some properties related to this path integral.

In this paper, we come back to the original map $\Psi$ of Wiener, by using Wiener chaos. But we use formal Wiener chaos. We consider a continuous path $w_{s}$. We consider a map $h_{n}^{i_{1}, \ldots, i_{n}}\left(s_{1}, \ldots, s_{n}\right) s_{1}<s_{2}<\cdots<s_{n}<1$ with values in $\mathbb{R}$. We consider the formal Wiener chaos:

$$
\Psi\left(h_{n}\right)=\int_{0<s_{1}<\cdots<s_{n}<1} h_{n}^{i_{1}, \ldots, i_{n}}\left(s_{1}, \ldots, s_{n}\right) d w_{s_{1}}^{i_{1}} \cdots d w_{s_{n}}^{i_{n}} .
$$

We put

$$
d w_{s}^{4}=24 d s
$$

If $i>4, d w_{s}^{i}=0$. We use in order to define the Wiener product on formal chaos associated to the Bilaplacian $L$ the Itô table for the Bilaplacian:

$$
d w_{s}^{i} d w_{s}^{j}=d w_{s}^{i+j}
$$


In order to simplify the exposition, we use in the sequel Connes space and not a Hida Fock space. We consider $L^{\infty}$ the set of map $h$ from $[0,1]$ into $\mathbb{R}^{3}$ such that

$$
\sup _{s}|h(s)|=\|h\|_{\infty} .
$$

We introduce the bosonic Connes space $\mathrm{CO}_{\infty-}\left(L^{\infty}\right)$ (a refinement of the traditional bosonic Fock space). To $\sigma \in C_{\infty}\left(L^{\infty}\right)$, we associate a formal Wiener chaos $\Psi(\sigma)$. We use the Itô table for the Bilaplacian in order to define a Wiener product on the bosonic Connes space:

$$
\Psi\left(\sigma_{1} \cdot \sigma_{2}\right)=\Psi\left(\sigma_{1}\right) \Psi\left(\sigma_{2}\right)
$$

The bosonic Connes space becomes a commutative topological algebra for the Wiener product (For similar consideration for the case of the standard Laplacian, we refer to the book of Meyer [3]).

We consider as classical the vacuum expectation on the bosonic Connes space, and we state a kind of Itô-Segal-Bargmann-Wiener isomorphism, but in this case there is no Hilbert space involved. We show that for the vacuum expectation $w_{s}$ has in some sense independent increments. We consider a type of generalization of the exponential martingale of the Brownian motion:

$$
\Psi\left(\sigma_{t}\right)=\sum \int_{0<s_{1}<\cdots<s_{n}<t} h_{s_{1}} d w_{s_{1}}^{1} \cdots h_{s_{n}} d w_{s_{n}}^{1}
$$

We suppose that $h$ is continuous. Let $f$ be a polynomial on $\mathbb{R}$. We put

$$
Q_{t}^{h}[f]=\mu\left[f\left(w_{t}^{1}\right) \Psi\left(\sigma_{t}\right)\right]
$$

We show the following Cameron-Martin-Maruyama-Girsanov type formula:

$$
\frac{\partial}{\partial t} Q_{t}^{h}[f]=Q_{t}^{h}\left[L_{h, t} f\right]
$$

where

$$
L_{h, t}=L+\text { lowerterm }
$$

\section{Formal Wiener Chaos Associated to a Bilaplacian}

We consider the set $L^{\infty} .\left(L^{\infty}\right)^{\otimes n}$ is constituted of maps:

$$
\sum_{i_{1}, \ldots, i_{n}} h^{i_{1}, \ldots, i_{n}}\left(s_{1}, \ldots, s_{n}\right) e_{i_{1}} \otimes \cdots \otimes e_{i_{n}}=h_{n}\left(s_{1}, \ldots, s_{n}\right),
$$

where $e_{i}$ is the standard basis of $\mathbb{R}^{3}$. On $\left(L^{\infty}\right)^{\otimes n}$, we consider the natural supremum norm $\left\|h_{n}\right\|_{\infty}$. Moreover, there is a natural action of the symmetric group on $\left(L^{\infty}\right)^{\otimes n}$. Elements which 
are invariant under this action of the symmetric group are called elements of the symmetric tensor product $\left(L^{\infty}\right)^{\widehat{\otimes} n} . C O_{C, r}\left(L^{\infty}\right)(r>0, C>0)$ is constituted of formal series $\sigma=\sum h_{n}$ where $h_{n}$ belongs to $\left(L^{\infty}\right)^{\widehat{\otimes} n}$ such that

$$
\|\sigma\|_{C}=\sum C^{n} n !\left\|h_{n}\right\|_{\infty}<\infty
$$

Definition 2.1. The intersection of all $\mathrm{CO}_{C}\left(L^{\infty}\right)$ is called the bosonic Connes space $\mathrm{CO}_{\infty-}\left(L^{\infty}\right)$.

Remark 2.2. In the sequel we could choose an Hida Fock space.

Definition 2.3. The vacuum expectation $\mu$ on $\mathrm{CO}_{\infty-}\left(L^{\infty}\right)$ is defined by

$$
\mu(\sigma)=h_{0} .
$$

If $h_{n}$ belongs to $\left(L^{\infty}\right)^{\hat{\otimes} n}$, we consider the formal Wiener chaos:

$$
\Psi\left(h_{n}\right)=\sum_{i_{1}, \ldots, i_{n}} \int_{0<s_{1}<\cdots<s_{n}<1} h_{n}^{i_{1}, \ldots, i_{n}}\left(s_{1}, \ldots s_{n}\right) d w_{s_{1}}^{i_{1}} \cdots d w_{s_{n}}^{i_{n}}
$$

We could do the same expression if $h_{n}$ belongs to $\left(L^{\infty}\right)^{\otimes n}$.

Definition 2.4. The map $\Psi$ defined on $\mathrm{CO}_{\infty-}\left(L^{\infty}\right)$ is called the map formal Wiener chaos.

Let $\{1, \ldots, n\},\{n+1, \ldots, n+m\}$. Let $\{l\}$ be a concatenation (or pairing). It is an increasing injective map from a set with $l$ element in $\{1, \ldots, n\}$ into $\{n+1, \ldots, n+m\}$. There is at most $C^{n+m}$ pairing of length $l$. We consider $h_{n}^{1} \otimes_{\{l\}, s h_{\{l}} h_{m}^{2}$ where we concatain the time in $h_{n}$ and in $h_{m}$ according the pairing, and we shuffle according to the shuffle $s h_{l}$ and the time in $h_{n}^{1}$ and $h_{m}^{1}$ between two continuous times in the pairing. When we concatenate two times, we use the Itô table for the Bilaplacian, and we symmetrized the expression in the time.

The classical product of $\Psi\left(h_{n}^{1}\right) \Psi\left(h_{m}^{2}\right)$ is equal to $\sum_{\{l\}, s h_{\{l\}}} \Psi\left(h_{n}^{1} \otimes_{\{l\}, s h_{\{l\}}} h_{m}^{2}\right)$ and generalized with this new Itô table the standard formula which gives the product of two Wiener chaos in the Brownian case. There are at most $C^{n+m} C_{n}^{l} C_{m}^{l}$ pairing $\{l\}$ and shuffle according to the pairing $\{l\}$.

Definition 2.5. The Wiener product of $h_{n}^{1}$ and $h_{m}^{2}$ is defined by

$$
\Psi\left(h_{n}^{1} \cdot h_{m}^{2}\right)=\Psi\left(h_{n}^{1}\right) \Psi\left(h_{n}^{2}\right)
$$

Theorem 2.6. The Wiener product endows the symmetric Connes space with a structure of topological commutative algebra.

Proof. Let us show first of all that the Wiener product is continuous. We have

$$
\left\|h_{n}^{1} \otimes_{\{l\}, s h\{l\}} h_{m}^{2}\right\|_{\infty} \leq C^{n+m}\left\|h_{n}^{1}\right\|_{\infty}\left\|h_{m}^{2}\right\|_{\infty}
$$


Therefore,

$$
\left\|h_{n}^{1} \cdot h_{m}^{2}\right\|_{C} \leq C_{1}^{n+m} C^{n+m}\left\|h_{n}^{1}\right\|_{\infty}\left\|h_{m}^{2}\right\|_{\infty} \sum_{\{l\}, s h_{\{l\}}} C^{-l}((n+m-2 l) !)
$$

But

$$
\sum_{\{l\}, s h_{[l]}} C^{-l} \leq \sum_{l} C_{n}^{l} C_{m}^{l} C_{3}^{n+m} C^{-l} \leq C_{2}^{n+m}\left(1+C^{-1}\right)^{n+m} \leq C_{4}^{n+m}
$$

On the other hand, by the Stirling formula,

$$
(n !)^{-1}(m !)^{-1}(n+m-2 l) ! \leq C_{3}^{n+m} .
$$

We deduce that

$$
\left\|\sigma_{1} \cdot \sigma_{2}\right\|_{C} \leq K\left\|\sigma_{1}\right\|_{C^{\prime}}\left\|\sigma_{2}\right\|_{C^{\prime}}
$$

and therefore the Wiener product is continuous on the bosonic Connes space.

Let $h_{n_{1}}, h_{n_{2}}$, and $h_{n_{3}}$ be 3 elements of the bosonic Connes space.

Let $s h_{1,2,3}$ be a shuffle between the 3 sets $\left\{1, n_{1}\right\},\left\{n_{1}+1, n_{1}+n_{2}\right\}$, and $\left\{n_{1}+n_{2}+\right.$ $\left.1, n_{1}+n_{2}+n_{3}\right\}$.

We perform two concatenations between the times when the shuffle is done:

(i) either we concatain 2 contiguous times in $\left\{1, n_{1}\right\}$ and in $\left\{n_{1}+1, n_{1}+n_{2}\right\}$ and two contiguous time in $\left\{1, n_{1}\right\}$ and in $\left\{n_{1}+n_{2}+1, n_{1}+n_{2}+n_{3}\right\}$;

(ii) either we concatain 2 contiguous times in $\left\{n_{1}+1, n_{1}+n_{2}\right\}$ and in $\left\{1, n_{1}\right\}$ and two contiguous times in $\left\{n_{1}+1, n_{1}+n_{2}\right\}$ and in $\left\{n_{1}+n_{2}+1, n_{1}+n_{2}+n_{3}\right\}$;

(iii) either we concatain 2 contiguous times in $\left\{n_{1}+n_{2}+1, n_{1}+n_{2}+n_{3}\right\}$ and in $\left\{1, n_{1}\right\}$ and two contiguous times in $\left\{n_{1}+n_{2}+1, n_{1}+n_{2}+n_{3}\right\}$ and in $\left\{n_{1}+1, n_{1}+n_{2}\right\}$;

(iv) or we concatain 3 contiguous times in $\left\{1, n_{1}\right\}$, in $\left\{n_{1}+1, n_{1}+n_{2}\right\}$ and in $\left\{n_{1}+n_{2}+\right.$ $\left.1, n_{1}+n_{2}+n_{3}\right\}$.

When we concatain time, we use the iterated Itô rule:

$$
\left(d w_{s}^{i_{1}} \cdot d w_{s}^{i_{2}}\right) \cdot d w_{s}^{i_{3}}=d w_{s}^{i_{1}+i_{2}+i_{3}}
$$

Such a concatenation is called $l_{1,2,3}$ and the final result is called $h_{n_{1}} \otimes_{s h_{1,2,3}, l_{1,2,3}} h_{n_{2}} \otimes_{s h_{1,2,3} l_{1,2,3}} h_{n_{3}}$. We deduce the formula

$$
\left(h_{n_{1}} \cdot h_{n_{2}}\right) \cdot h_{n_{3}}=\sum_{l_{1,2,3}, s h_{1,2,3}} h_{n_{1}} \otimes_{s h_{1,2,3}, l_{1,2,3}} h_{n_{2}} \otimes_{s} h_{1,2,3} l_{1,2,3} h_{n_{3}} .
$$

From this formula we deduce the associativity of the Wiener product.

From the product formula, we deduce easily the next theorem. 
Theorem 2.7 (Itô-Bargmann-Wiener-Segal). Let $h_{n_{1}}^{i_{1} \ldots, i_{n_{1}}}$ and $h_{n_{2}}^{j_{1} \ldots, j_{n_{2}}}$ be elements of the bosonic Connes space. They are seen as a function on the involved simplices. Then

$$
\begin{aligned}
\mu\left[\Psi\left(h_{n_{1}}\right) \Psi\left(h_{n_{2}}\right)\right]= & \delta_{n_{1}, n_{2}} \prod \delta_{i_{l}+j_{l}=4} 24^{n_{1}} \\
& \times \int_{0<s_{1}<\cdots<s_{n}<1} h_{n_{1}}^{i_{1}, \ldots, i_{n}}\left(s_{1}, \ldots, s_{n}\right) h_{n_{1}}^{j_{1}, \ldots, j_{n}}\left(s_{1}, \ldots, s_{n}\right) d s_{1} \cdots d s_{n} .
\end{aligned}
$$

Remark 2.8. In the case of the classical Laplacian, this formula justifies the choice of $\mathbb{H}$ instead of $L^{\infty}$. But in the previous formula, only a prehilbert space appears. So it is not obviously justified to choose $\mathbb{H}$ instead of $L^{\infty}$ to perform our computations. We have chosen $L^{\infty}$ because the estimates are simpler with this space.

We say that $h_{n}$ belongs to $C O_{\infty-, t]}\left(L^{\infty}\right)$ if $h_{n}$ vanishes as soon as one of the $s_{i} \geq t$. We say that $h_{n}$ belongs to $C O_{\infty-,[t}\left(L^{\infty}\right)$ if $h_{n}$ vanishes as soon as one of the $s_{i} \leq t$. We get the next theorem whose proof is obvious.

Theorem 2.9. $C \mathrm{CO}_{\infty-, t]}\left(L^{\infty}\right)$ and $C \mathrm{CO}_{\infty-,[t}\left(L^{\infty}\right)$ are subalgebras of $C \mathrm{O}_{\infty-}\left(L^{\infty}\right)$ for the Wiener product. Moreover, if $\sigma_{1} \in C \mathrm{C}_{\infty-, t]}\left(L^{\infty}\right)$ and if $\sigma_{2} \in \mathrm{CO}_{\infty-,[t}\left(L^{\infty}\right)$,

$$
\mu\left[\Psi\left(\sigma_{1}\right) \Psi\left(\sigma_{2}\right)\right]=\mu\left[\Psi\left(\sigma_{1}\right)\right] \mu\left[\Psi\left(\sigma_{2}\right)\right]
$$

Remark 2.10. Let us justify heuristically this part. Let $Q_{t}^{0}$ be the semi-group generated by $L$. Let us suppose that there is a formal measure $d \mu$ on a path space $t \rightarrow w_{t}$ such that

$$
Q_{t}^{0}[f]=\int f\left(w_{t}\right) d \mu
$$

(In the case of the standard Laplacian it is the measure of the Brownian motion). We refer to [19] for a physicist way to construct this measure. We have

$$
Q_{t}^{0}\left[x^{4}\right]=24 t
$$

So the infinitesimal increment $\left(d w_{t}\right)^{i}$ of $w_{t}$ should satisfy the Itô table (1.12) and the formal Wiener chaos should be an extension of the classical Wiener chaos in the Brownian case.

\section{A Cameron-Martin-Maruyama-Girsanov Formula Associated to a Bilaplacian}

We put if $f$ is a polynomial,

$$
Q_{t}^{h}[f]=\mu\left[f\left(w_{t}^{1}\right) \Psi\left(\sigma_{t}\right)\right]
$$


where

$$
\Psi\left(\sigma_{t}\right)=\sum \int_{0<s_{1}<\cdots<s_{n}<t} h_{s_{1}} d w_{s_{1}}^{1} \cdots h_{s_{n}} d w_{s_{n}}^{1}
$$

We suppose that $h$ is continuous. In this formula, only finite sums appear due to (2.13). We get the following.

Theorem 3.1 (Cameron-Martin-Maruyama-Girsanov). If $f$ is a polynomial,

$$
\frac{\partial}{\partial t} Q_{t}^{h}[f]=Q_{t}^{h}\left[L_{h, t} f\right]
$$

where

$$
L_{h}=-\frac{\partial^{4}}{\partial x^{4}}+\alpha h_{t} \frac{\partial^{3}}{\partial x^{3}}
$$

Proof. Let us consider the case where $f(x)=x^{n}$. We use $w_{t}^{1}=\int_{0}^{t} d w_{s}^{1}$ and the fact that the Wiener product is associative. We get

$$
\left(w^{1}+w_{t+\Delta t}^{1}-w_{t}^{1}\right)^{n}=\sum C_{n}^{k}\left(w_{t}^{1}\right)^{n-k}\left(w_{t+\Delta t}^{1}-w_{t}^{1}\right)^{k}
$$

We put

$$
\sigma_{\Delta t}=\sum \frac{\mathbb{I}_{[t, t+\Delta t]}^{\otimes n}}{n !}
$$

such that by the Itô rules on $[t, t+\Delta t]$ for $\Delta t>0$ :

$$
\sigma_{t+\Delta t}=\sigma_{t} \cdot \sigma_{\Delta_{t}}
$$

We use Theorem 2.9 and the Itô table on $[t, t+\Delta t]$. We deduce that

$$
\begin{aligned}
\mu\left[\left(w_{t+\Delta t}^{1}\right)^{n} \Psi\left(\sigma_{t+\Delta t}\right)\right]= & \mu\left[\left(w_{t}^{1}\right)^{n} \Psi\left(\sigma_{t}\right)\right] \\
& +n(n-1)(n-2)(n-3) \mu\left[\left(w_{t}^{1}\right)^{n-4} \Psi\left(\sigma_{t}\right)\right] \Delta t \\
& +\alpha h_{t} n(n-1)(n-2) \mu\left[\left(w_{t}^{1}\right)^{n-3} \Psi\left(\sigma_{t}\right)\right] \Delta_{t}+o(\Delta t) .
\end{aligned}
$$

Therefore, the result is obtained. 


\section{References}

[1] T. Hida, H.-H. Kuo, J. Potthoff, and L. Streit, White Noise: An Infinite-Dimensional Calculu, vol. 253, Kluwer Academic Publishers, Dordrecht, The Netherlands, 1993.

[2] N. Obata, White Noise Calculus and Fock Space, vol. 1577 of Lecture Notes in Mathematics, Springer, Berlin, Germany, 1994.

[3] P.-A. Meyer, Quantum Probability for Probabilists, vol. 1538 of Lecture Notes in Mathematics, Springer, Berlin, Germany, 1993.

[4] R. Léandre, "Itô-Stratonovitch for a four order operator on a torus," in Non-Euclidean Geometry and Its Applications, P. Nagy and P. Raics, Eds., vol. 42 of Acta Physica Debrecina, pp. 133-138, 2008.

[5] R. Léandre, "Itô-Stratonovitch for the Schroedinger equation associated to a big order operator on a torus," in Fractional Order Differentiation, G. Zaslavsky, D. Baleanu, and J. A. Tenreiro Machado, Eds., vol. 136 of Physica Scripta, p. 014028, 2009.

[6] R. Léandre, "Itô-Stratonovitch formula for the wave equation on a torus," in Computations of Stochastic Systems, M. A. El Tawil, Ed., vol. 5890 of LNCS Transactions on Computational Science, pp. 68-75, 2010.

[7] R. Léandre, "Itô formula for an integro differential operator without a stochastic process," in Proceedings of the 20th International Symposium on Algorithms and Computation (ISAAC '09), M. Ruzhansky and J. Wirth, Eds., pp. 225-231, World Scientific, 2009.

[8] R. Léandre, "Stochastic analysis without probability: study of some basic tools," Journal of PseudoDifferential Operators and Applications, vol. 1, no. 4, pp. 389-410, 2010.

[9] R. Leandre, "Long time behaviour on a path group of the heat semi-group associated to a bilaplacian," Symmetry, vol. 3, no. 1, pp. 72-83, 2011.

[10] R. Léandre, "The Itô transform for an accretive operators," Axioms, vol. 1, pp. 4-8, 2012.

[11] T. Funaki, "Probabilistic construction of the solution of some higher order parabolic differential equation," Proceedings of the Japan Academy A, vol. 55, no. 5, pp. 176-179, 1979.

[12] K. J. Hochberg, "A signed measure on path space related to Wiener measure," The Annals of Probability, vol. 6, no. 3, pp. 433-458, 1978.

[13] V. Ju. Krylov, "Some properties of the distribution corresponding to the equation $\partial u / \partial t=$ $(-1)^{q+1} \partial^{2 q} u / \partial x^{2 q}$," Doklady Akademii Nauk SSSR, vol. 132, pp. 1254-1257, 1960, Soviet Mathematics. Doklady, vol. 1, pp. 760-763, 1960.

[14] S. Mazzucchi, "Probabilistic representations for the solution of higher order differential equations," University Torino, 2011.

[15] S. Albeverio, "Wiener and Feynman-path integrals and their applications," in Proceedings of the Norbert Wiener Centenary Congress, V. Mandrekar and P. R. Masani, Eds., vol. 52 of Proceedings of Symposia in Applied Mathematics, pp. 163-194, American Mathematical Society, Providence, RI, USA, 1996.

[16] E. Getzler, "Cyclic homology and the path integral of the Dirac operator," In press.

[17] R. Léandre, "Connes-Hida calculus in index theory," in Proceedings of the 14th International Congress on Mathematical Physics, J. C. Zambrini, Ed., pp. 493-497, World Scientific, 2004.

[18] R. Léandre, "Path integrals in noncommutive geometry," in Encyclopedia of Mathematical Physics, G. Naber and T. S. Tsun, Eds., pp. 8-12, Elsevier, Oxford, UK, 2006.

[19] J. R. Klauder and S. V. Shabanov, "An introduction to coordinate free quantization and its application to constrained systems," in Mathematical Methods of Quantum Physics, C. C. Bernido, M. V. CarpioBernido, K. Nakamura, and K. Watanabe, Eds., pp. 117-130, Gordon and Breach, Amsterdam, The Netherland, 1999. 


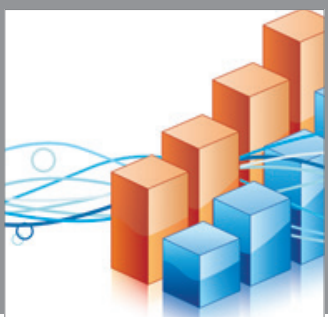

Advances in

Operations Research

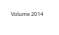

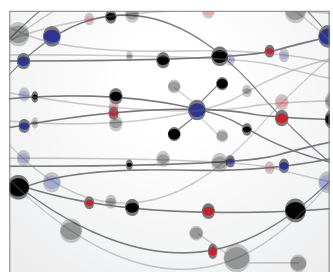

\section{The Scientific} World Journal
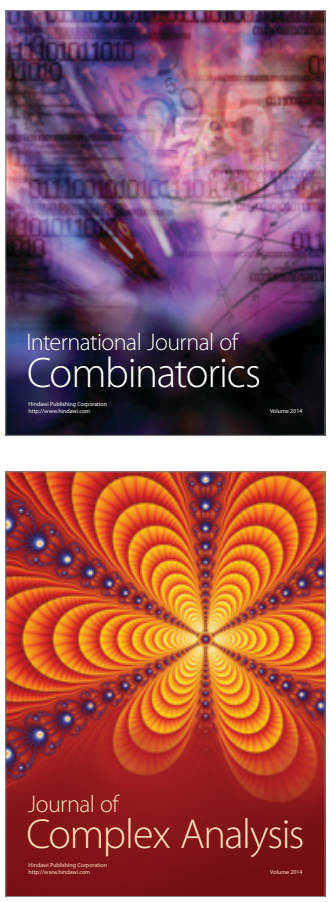

International Journal of

Mathematics and

Mathematical

Sciences
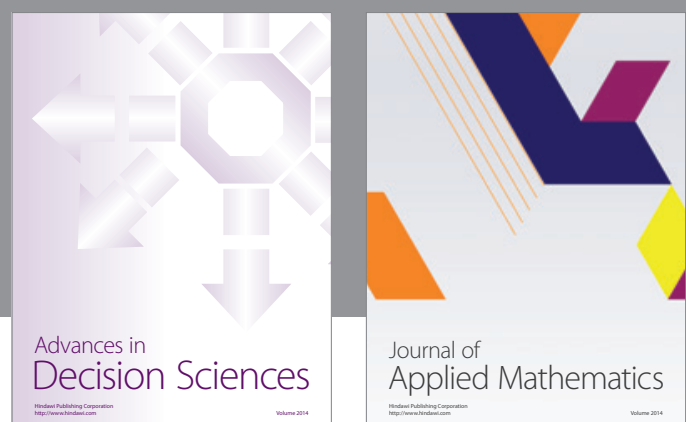

Journal of

Applied Mathematics
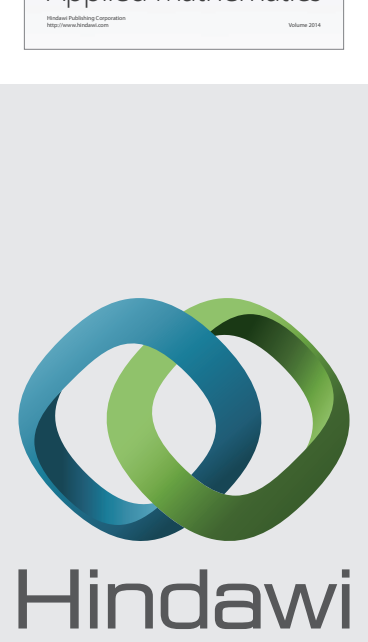

Submit your manuscripts at http://www.hindawi.com
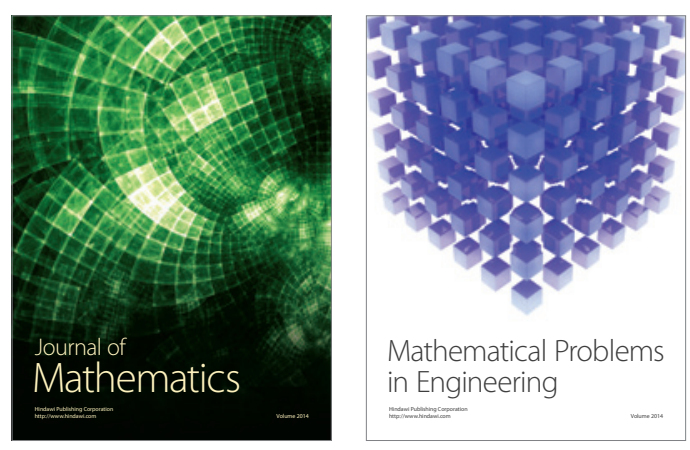

Mathematical Problems in Engineering
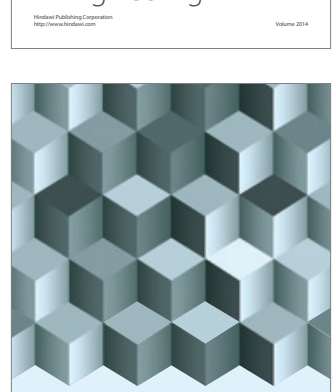

Journal of

Function Spaces
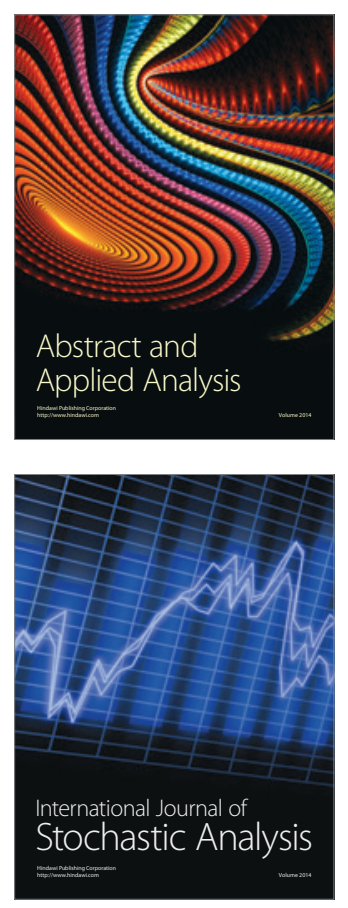

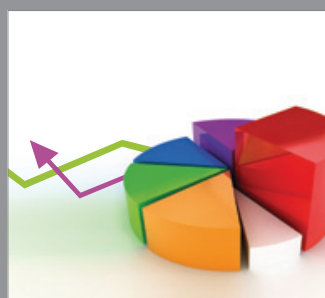

ournal of

Probability and Statistics

Promensencen
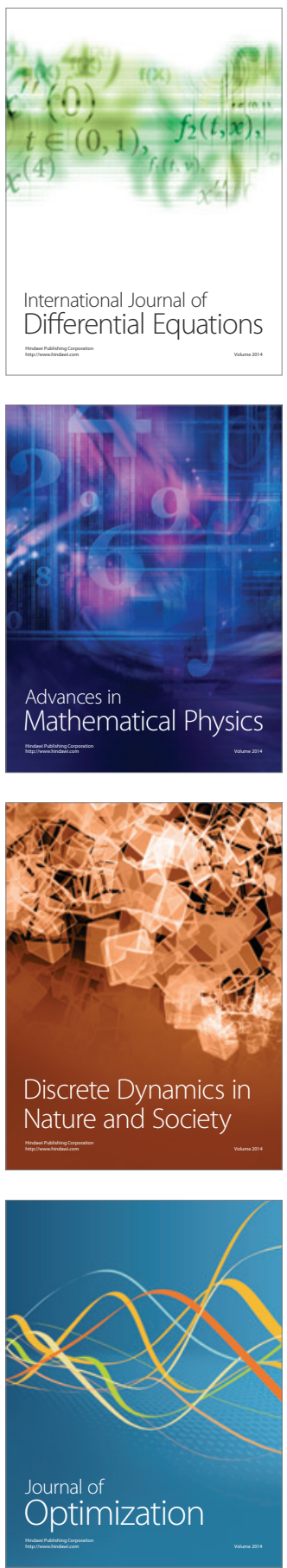